\title{
Enfoque Pediátrico para el Estudio de los Trastornos Respiratorios del Sueño
}

\author{
DANIEL ZENTENO A. ${ }^{1,2,}$, PAMELA SALINAS F. ${ }^{3}$, ROBERTO VERA U. ${ }^{4}$, \\ PABLO BROCKMANN V. ${ }^{5}$, FRANCISCO PRADO A..$^{3,6}$ \\ 1. Profesor Asistente, Departamento de Pediatría y Cirugía Infantil Sur, Facultad de Medicina, Universidad de Chile, \\ Santiago de Chile. Hospital Exequiel González Cortés. \\ 2. Unidad de Cuidados Especiales, Instituto Nacional de Rehabilitación, Pedro Aguirre Cerda. \\ 3. Unidad de Niños y Adolescentes con Necesidades Especiales en Salud, Servicio de Pediatría, Hospital Clínico \\ San Borja Arriarán. \\ 4. Programa Asistencia Ventilatoria No Invasiva, Ministerio de Salud de Chile. \\ 5. Instructor Asociado, Departamento de Pediatría, Pontificia Universidad Católica de Chile. Residente de Medicina del \\ Sueño, Universidad de Tübingen, Alemania. \\ 6. Profesor Asistente, Departamento de Pediatría Campus Centro, Facultad de Medicina, Universidad de Chile, \\ Santiago de Chile.
}

\begin{abstract}
\section{Pediatric Approach to Sleep Disorders Studies in Children}

Sleep Disorders can generate significant impact in the health of children and adolescents at a highly vulnerable period for neurodevelopment. A proper history and physical exam can lead the diagnosis, however there are significant limitations especially in the early phases of illness. Children with specific risks require a high level of suspicion, and early diagnostic studies. This review describes various diagnostic alternatives, feasible in different clinical situations, during sleep or wakefulness. These diagnostic studies may be useful in detection and early treatment of Sleep Disorders.
\end{abstract}

(Key words: Sleep Disorders Studies, polysomnography, Polygraphy, nocturnal Saturometry, non-invasive ventilation).

Rev Chil Pediatr 2010; 81 (5): 445-455

\section{RESUMEN}

Los Trastornos Respiratorios del Sueño (TRS) pueden generar un impacto en la salud de niños y adolescentes, en un período altamente vulnerable para el neurodesarrollo. La anamnesis y examen fisco pueden orientar a su diagnóstico, sin embargo, poseen limitaciones importantes, especialmente en etapas precoces de la enfermedad. En niños con factores de riesgo específicos es necesario tener un alto nivel de sospecha y realizar estudios diagnósticos. Este artículo de revisión tiene como objetivo describir las distintas alternativas diagnósticas, practicables en distintos escenarios clínicos y realizadas durante el sueño o vigilia. Estos métodos diagnósticos pueden ser de utilidad en el reconocimiento y tratamiento precoz de los TRS.

(Palabras clave: Estudios de sueño, Polisomnografía, Poligrafía, Saturometría nocturna, Ventilación No Invasiva).

Rev Chil Pediatr 2010; 81 (5): 445-455

Trabajo recibido el 17 de febrero de 2010, devuelto para corregir el 30 de marzo de 2010, segunda versión el 22 de abril de 2010, tercera versión el 24 de junio de 2010, aceptado para publicación el 17 de agosto de 2010.

Correspondencia a:

Dr. Daniel Zenteno A.

E-mail: danielzenteno@gmail.com 


\section{Introducción}

Los Trastornos Respiratorios del Sueño (TRS) son subdiagnósticados en población adulta y pediátrica. Incluyen un abanico de entidades respiratorias que conforman parte de un continuo, manifestándose y/o agravándose cuando el niño está durmiendo; pueden asociase a alteraciones en intercambio de gases, de arquitectura del sueño, apneas y/o hipoventilación $^{1,2}$. Dentro de estos se incluyen el Roncador Primario, Síndrome de Resistencia de Vía Aérea Superior, Hipoventilación Obstructiva y Síndrome de Apnea Obstructiva del Sueño (SAOS). La tabla 1 muestra la caracterización que las define ${ }^{3,4}$. Los TRS obedecen a una serie de causas que se clasifican en 3 grandes grupos: hipertofia adenotonsilar sin obesidad, obesidad y un último grupo donde se incluyen: Enfermedades Neuromusculares (ENM), Síndrome de Down, Parálisis Cerebral y Síndromes Craneofaciales ${ }^{5}$ (tabla 2).

En la población pediátrica general, la prevalencia del ronquido es de 3 a $12 \%$ y de SAOS 0,7 a $2 \%$, principalmente asociado a hipertrofia adenotonsilar ${ }^{2,6,7}$. En niños y ado- lescentes con ENM, obesidad o malformaciones craneofaciales, los TRS con significación clínica e impacto en el neurodesarrollo tienen una sustantiva mayor prevalencia, que incluso puede ser mayor al $50 \%{ }^{8}$.

Si bien existen síntomas que se han asociado a los TRS (tabla 3), la historia clínica y la anamnesis dirigida tienen una baja sensibilidad y/o especifidad, por lo que resulta necesario el apoyo con exámenes complementarios para llegar a un diagnóstico precoz, realizar una intervención oportuna y disminuir así la posibilidad de generar alteraciones neurocognitivas y efectos biológicos deletéreos en diversos sistemas como son metabólicos, cardiovascular y otros, con posibilidades de impactar en la calidad de vida relacionada a salud a lo largo de la vida ${ }^{9-11}$.

Los estudios de sueño se pueden subdividir, en forma no excluyente, en estudios: 1) Nocturnos; 2) Diurnos y 3) Dirigidos a niños con necesidades especiales ${ }^{11}$. La tabla 4 resume estas formas de evaluación del sueño y su escenario de realización, en orden de sensibilidad, calidad y cantidad de información entregada.

En el presente artículo se describen estas

Tabla 1. Caracterización de los Trastornos Respiratorios del Sueño

\begin{tabular}{|lccc|}
\hline & Roncadores primarios & $\begin{array}{c}\text { Resistencia aumentada } \\
\text { VAS }\end{array}$ & $\begin{array}{c}\text { Apnea Obstructiva del } \\
\text { Sueño }\end{array}$ \\
\hline Ronquido* & ++ & ++ & ++ \\
\hline Intercambio gaseoso & No & No & Sí \\
Signos de aumento RVAS & No & Sí & Sí \\
Alteración arquitectura sueño & $?$ & Sí & Sí \\
Consecuencias & Sí & Sí & Sí \\
\hline
\end{tabular}

* La intensidad del ronquido no define el riesgo de TRS. Los TRS suelen estar presentes con mayor frecuencia en niños con ronquido habitual, 3 o más noches/ emana. RVAS: Resistencia vía aérea superior.

Tabla 2. Clasificación de causas de los Trastornos Respiratorios del Sueño

\begin{tabular}{|lll|}
\hline TRS tipo I & TRS Tipo II & TRS Tipo III \\
\hline Hipertrofia adenotonsilar sin & Obesidad, con leve a moderado & Enfermedades neuromusculares \\
obesidad & Sipertrofia adenotonsilar & S Down \\
& & Arnold Chiari \\
& Paralisis cerebral \\
& Síndromes craneofaciales: Pierre Robin, Apert, Goldenhar, \\
& Crouzon, Acondroplasia \\
\hline
\end{tabular}


Tabla 3. Signos y síntomas asociados a Trastornos Respiratorios del Sueño

\begin{tabular}{|lll|}
\hline Síntomas & & Signos \\
Vigilia & Sueño & \\
Mal rendimiento escolar & Ronquido & Fascie adenoidea \\
Somnolencia & Apnea constatada por padres & Respirador bucal \\
Agresividad & Respiración paradojal & Aumento tejido amigdaliano \\
Hiperactividad & Enuresis & Alteraciones craneofaciales \\
Cefalea matinal & Sueño no reparador & Micrognatia \\
\hline Trastornos aprendizaje & & Alteración paladar \\
& & Hipertensión arterial \\
& & Hipocratismo digital \\
\hline
\end{tabular}

Tabla 4. Herramientas para el estudio de Trastornos Respiratorios del Sueño con sus respectivos escenarios de realización y/o interpretación

\begin{tabular}{|c|c|}
\hline Estudios nocturnos & Lugar de realización y/o interpretación \\
\hline $\begin{array}{l}\text { a. Polisomnografía } \\
\text { b. Poligrafía } \\
\text { c. Saturometría nocturna }\end{array}$ & $\begin{array}{l}\text { Hospitalaria } \\
\text { Domiciliaria preferentemente, hospitalaria ocasionalmente } \\
\text { Domiciliaria preferentemente, hospitalaria ocasionalmente }\end{array}$ \\
\hline \multicolumn{2}{|l|}{ Estudios diurnos } \\
\hline $\begin{array}{l}\text { a. Gases en sangre } \\
\text { b. Fuerza muscular respiratoria } \\
\text { c. Espirometría } \\
\text { d. Capnografía volumétrica }\end{array}$ & $\begin{array}{l}\text { Preferentemente hospitalario, domiciliario programado u ocasional } \\
\text { Domiciliario o policlínico de especialidad } \\
\text { Domiciliario o policlínico de especialidad } \\
\text { Domiciliario }\end{array}$ \\
\hline \multicolumn{2}{|l|}{ Estudios en niños con necesidades especiales } \\
\hline $\begin{array}{l}\text { a. Lectura de tarjetas de memoria de ventiladores mecánicos } \\
\text { b. Lectura de tarjetas de memoria de monitores de Apnea }\end{array}$ & $\begin{array}{l}\text { Domiciliaria o en policlínico de especialidad } \\
\text { Domiciliaria o en policlínico de especialidad }\end{array}$ \\
\hline
\end{tabular}

distintas alternativas para el diagnóstico y seguimiento de niños y adolescentes con TRS, poniendo énfasis en aspectos prácticos de evaluación a través de modelos de estudio que combinen acciones diagnósticas escalonadas ambulatorias y/o con internaciones abreviadas por una o dos noches, realizadas en forma protocolizadas y progresivas, destinadas a garantizar la pesquisa de los TRS, optimizando eficacia y calidad, con la mayor cobertura posible otorgada a la población en riesgo.

\section{Estudios nocturnos}

Son evaluaciones realizadas durante el sueño y reconocidas en diferente grado, para el estudio de TRS. Su grado de precisión diagnóstica va en descenso, es decir desde la polisomnografía a poligrafía y saturometría nocturna ${ }^{12-14}$.

\section{a. Polisomnografía}

La Polisomnografía (PSG) es el estándar de oro para estudiar sueño, nos entrega información completa, que incluye principalmente parámetros neurofisiológicos y cardiorrespiratorios. Integra en un registro continuo en línea y simultáneo electroencefalografía, electromiografía, electrooculografía, electrocardiografía, saturación de oxígeno $\left(\mathrm{SpO}_{2}\right)$, banda de esfuerzo torácico y abdominal, sensor de posición, sensor de flujo naso-bucal por algoritmo de presión, sensor de flujo aéreo naso-bucal 
por algoritmo de temperatura, micrófono, sensor de luz ambiental, capnografía exhalatoria $\left(\mathrm{ETCO}_{2}\right)$ (opcional), registro de video (opcional) y medición de presiones en la vía aéreainterfase en caso de titulación de asistencia ventilatoria $^{12,15}$ (figura 1).

Sus principales desventajas son que requiere de la internación del paciente, instalaciones adecuadas para un buen dormir, un equipo capacitado en la realización e interpretación del examen; consecuentemente es de alto costo ${ }^{15}$. Actualmente existen posibilidades restringidas para la realización de este examen en el sistema público de nuestro país, situación descrita también en países desarrollados.

En niños y adolescentes hay consideraciones para la lectura de eventos respiratorios entregadas por la Academia Americana de Medicina del Sueño (AASM) ${ }^{16}$, que se resumen en la tabla 5. Estas han sido propuestas para pacientes $\leq$ de 18 años, sin embargo, excepcionalmente, desde los 13 años podrían ser utilizadas recomendaciones establecidas para adultos $^{16}$.

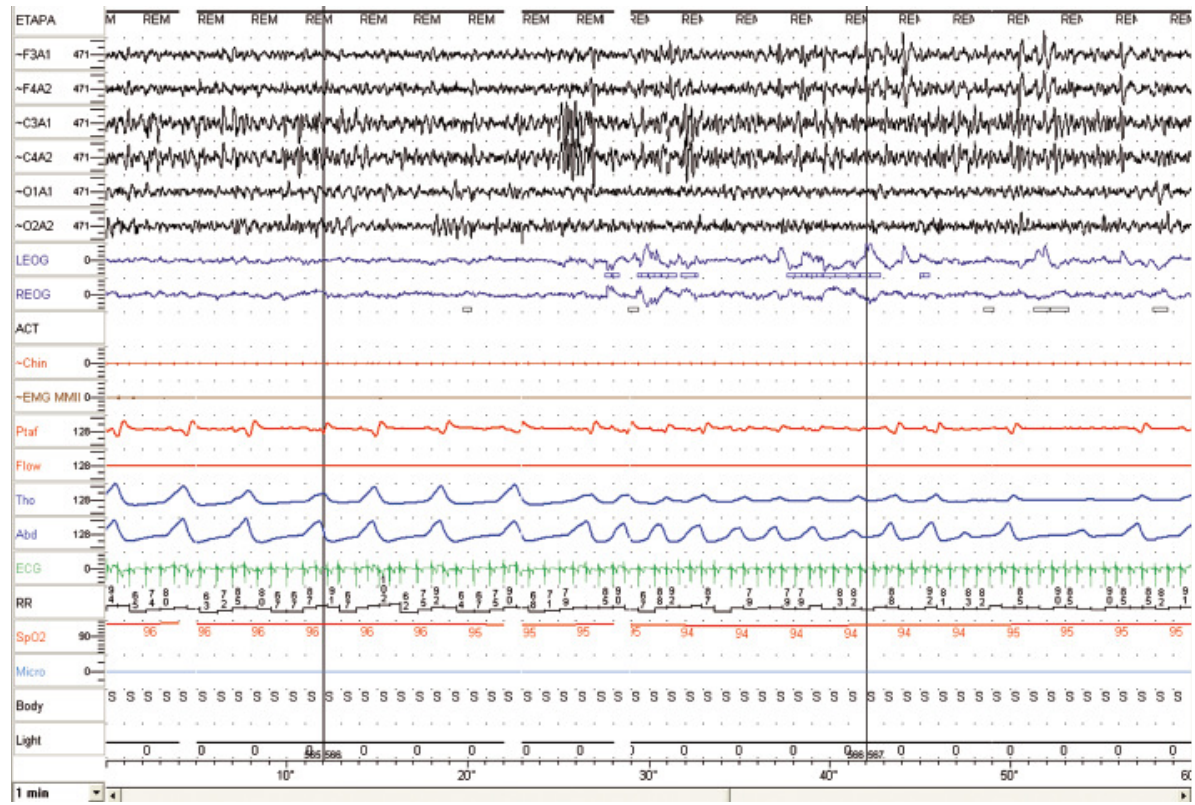

Figura 1. Trazado polisomnográfico en sueño REM, sin eventos respiratorios.

Tabla 5. Consideraciones para lectura de eventos respiratorios en PSG, en pacientes $\leq$ de 18 años ${ }^{16}$

\begin{tabular}{|c|c|}
\hline Apnea obstructiva & $\begin{array}{l}\text { Ausencia o reducción de flujo naso-bucal } \geq 90 \% \text {, en más de } 2 \text { ciclos respiratorios, con mantención o signos } \\
\text { de esfuerzo inspiratorio (ej: Respiración paradojal) }\end{array}$ \\
\hline Apnea central & $\begin{array}{l}\text { Se deben cumplir al menos } 1 \text { de las siguientes: } \\
\text { a. Ausencia de flujo naso-bucal, de } \geq 20 \text { segundos de duración, sin esfuerzo inspiratorio } \\
\text { b. Ausencia de flujo naso-bucal, en más de } 2 \text { más ciclos respiratorios, sin esfuerzo inspiratorio; asociado a } \\
\text { despertar, microdespertar o desaturación } \geq 3 \% \text { del basal }\end{array}$ \\
\hline Apnea mixta & $\begin{array}{l}\text { Ausencia o reducción de flujo naso-bucal } \geq 90 \% \text {, en más de } 2 \text { ciclos respiratorios, sin esfuerzo inspiratorio } \\
\text { en porción inicial del evento, seguido de recuperación de este esfuerzo antes del final del evento }\end{array}$ \\
\hline Hipopnea & $\begin{array}{l}\text { Reducción de la amplitud del flujo oronasal en } \geq 50 \% \text {, por más de } 2 \text { ciclos respiratorios, medido con PTAF. } \\
\text { Debe asociarse a despertar, microdespertar o desaturación } \geq 3 \% \text { del basal }\end{array}$ \\
\hline Hipoventilación & Registros de $\mathrm{ETCO}_{2}>50 \mathrm{mmHg},>25 \%$ del TTS \\
\hline Respiración periódica & $\begin{array}{l}\text { > de } 3 \text { episodios de apnea central, de más de } 3 \text { segundos de duración, separados por menos de } 20 \\
\text { segundos de respiración normal }\end{array}$ \\
\hline
\end{tabular}

PTAF: Sensor de flujo naso-bucal por algoritmo de presión. ETCO : Capnografia exhalatoria. TTS: Tiempo total del sueño. 
Se requiere de personal entrenado en la instalación de los canales y lectura del software del polisomnógrafo para evaluar la calidad de la señal mientras se realiza el examen, asegurando un buen registro. En el caso del análisis se requiere de un mínimo de $4 \mathrm{~h}$ de tiempo total de sueño (TTS) $(>2$ ciclos de sueño REM), es recomendable realizar interpretación manual de los eventos por profesionales con experiencia en trastornos neurofisiológicos y respiratorios del sueño ${ }^{17}$.

La PSG debiera complementarse con una medición de $\mathrm{CO}_{2}$ (ej: $\mathrm{ETCO}_{2}$ continua), integrada al resto de los canales, en especial frente a sospecha de TRS en su etapa inicial. Para determinar presencia de hipoventilación, algunos autores proponen puntos de corte más bajos a los establecido por la AASM, categorizando el grado de hipoventilación según el porcentaje de TTS sobre $50 \mathrm{mmHg}$; leve entre $10-24 \%$, moderado $25-49 \%$ y severo $>50 \%{ }^{18}$.

\section{b. Poligrafia}

La poligrafía ( $\mathrm{PG}$ ) clásica consiste en el análisis de variables cardiorrespiratorias sin evaluar los parámetros neurofisiológicos del sueño. Integra en un registro en línea y simultáneo la monitorización continua de electrocardiografía, $\mathrm{SpO}_{2}$, banda de esfuerzo torácica y abdominal o sólo torácica, sensor de posición y sensor de flujo nasal por algoritmo de presión o sensor de flujo aéreo naso-bucal por algoritmo de temperatura y sensor de ronquido ${ }^{19}$.

Algunos de ellos pueden ser conectados en línea a los dispositivos generadores de flujo, Cpap y Bipap, permitiendo medir la presión generada en la vía aérea, volumen corriente y flujo de escape. Otros permiten, a través de un adaptador puesto en línea en el circuito monorama (corrugado) de los generadores de flujo, medir la señal de flujo por medio de un sensor de presión; además algunos poseen canales auxiliares para $\mathrm{ETCO}_{2}$. Estas alternativas se han diseñado para ser de ayuda en el diagnóstico, titulación de los equipos para asistencia ventilatoria no invasiva y su seguimiento en domicilio $^{20}$ (figura 2).

La PG tiene un menor costo y puede ser realizada en el domicilio lo que favorece que el patrón de sueño durante el estudio se asimile más al patrón habitual del sueño del pacien$\mathrm{te}^{13}$. En adultos su utilidad para diagnóstico de SAOS está claramente demostrada, describiéndose alta concordancia con hallazgos polisomnográficos al ser comparada con poligrafía de lectura domiciliaria ${ }^{21}$. En población pediátrica, si bien la experiencia es escasa, esta validada para el diagnóstico de SAOS, encontrándose concordancia de un $85 \%$ con respecto a la $\mathrm{PSG}^{22}$. Existen recientes publicaciones que proponen valores de referencia en escolares, estos son similares a los propuestos para eventos respiratorios polisomnográficos ${ }^{13,19}$. Su aplicación podría ser de gran interés para el estudio de los TRS en niños roncadores y en población de pacientes con ENM y/o SAOS tipo 2 y $3^{5,9}$.

$\mathrm{Al}$ igual que la PSG, se requiere la instalación por personal entrenado, la lectura del software del polígrafo para evaluar la calidad de la señal mientras se realiza el examen es variable dependiendo del lugar de realización (domicilio o institucional). Para el análisis se requiere de un mínimo de $4 \mathrm{~h}$ del TTS, se recomienda utilizar la interpretación manual de los eventos por profesionales capacitados en los TRS ${ }^{12,20}$. Los índices de los eventos respiratorios se expresan según el tiempo total de registro y no tiempo total de sueño.

\section{c. Saturometría nocturna}

La oximetría de pulso es un método validado, seguro y frecuentemente usado para la detección de la hipoxemia crónica y/o la hipoxemia intermitente ${ }^{8}$. Su uso ha ido en aumento en la evaluación de los TRS, debido a su bajo costo, fácil uso, y la capacidad de entregar información precisa ${ }^{14}$.

Este examen usualmente se realiza en el domicilio del paciente. Al igual que la PSG y PG, la saturometría nocturna debe contar con instalaciones que proporcionen un ambiente grato con las comodidades para dormir (zona tranquila, sin ruidos, poca luz, ideal con cama única y evitar elementos eléctricos como radios, computadores y televisores) y a la vez seguro para poder realizar la instalación del equipo $^{12,15}$.

Todos los monitores de $\mathrm{SpO}_{2}$ presentan 


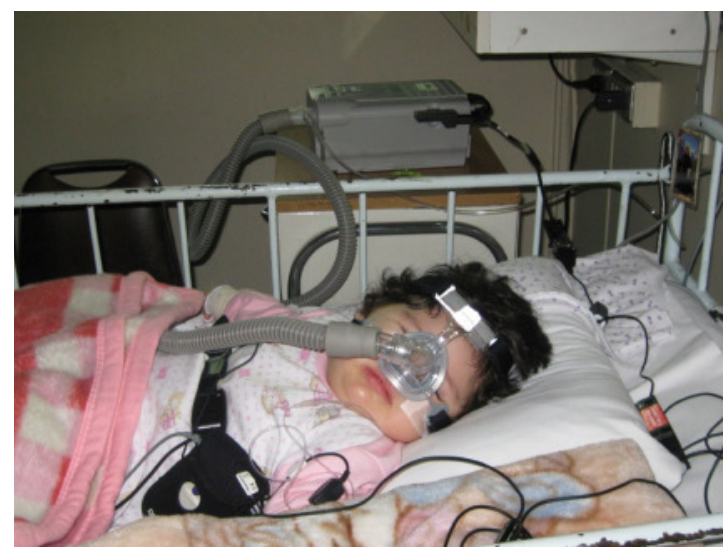

Figura 2A. Imagen de lactante en estudio poligráfico, en proceso de titulación para equipo de Asistencia Ventilatoria No Invasiva (AVNI).

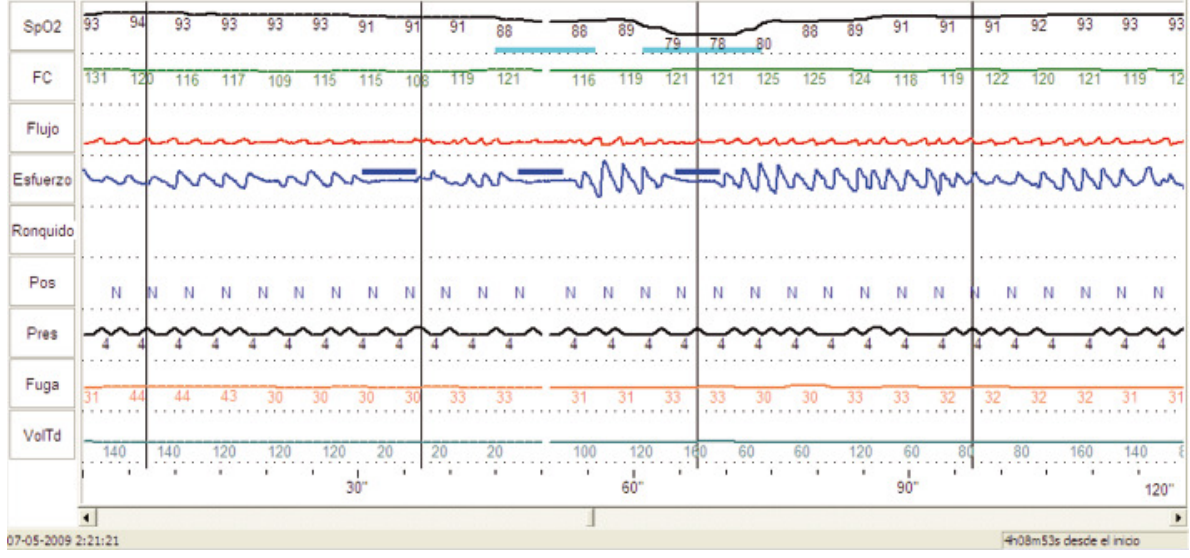

Figura 2B. Imagen de software de poligrafía incluyendo instalación de AVNI. Se observan apneas centrales asociadas a desaturaciones hasta $78 \%$ en un lactante con Síndrome de Arnold Chiari tipo 2 y mielomeningocele operado. Se instalo AVNI con soporte (frecuencia respiratoria). cierta variabilidad entre sus mediciones y con los resultados reales de la $\mathrm{SpO}_{2}$ medida con un cooxímetro. La tecnología de señal de extracción digital y su software, de interpretación con lectura promediada cada dos segundos, han mejorado su umbral de medición en presencia de artefactos de movimiento, ruido y mala perfusión. Diversos estudios de la tecnología de los oximetros de pulso demuestran que la tecnología de los equipos Masimo es superior en el estudio de oximetría simple y continua; disminuye el porcentaje de falsas alarmas, fenómenos no percibidos y artefac$\operatorname{tos}^{23,24}$ (figura 3).

$\mathrm{El}$ análisis de la saturometría nocturna debe ser informado por profesional entrenado en el software y con formación en TRS. Si bien los softwares de los distintos equipos de saturo- metría continua permiten obtener un informe del tiempo de registro, promedio de saturación, promedio de frecuencia cardíaca, $\%$ del tiempo de registro con $\mathrm{SpO}_{2}<90 \%$, índice de eventos de desaturación mayor o igual al 4\%, gráficos e histogramas del análisis; es necesario realizar lectura del registro eliminando artefactos y tiempos que según registro del cuidador puedan llevar a errores en el análisis ${ }^{14,24}$. Ej: se despierta para ir al baño, se despierta para alimentarse, etc.

Se ha comparado con la PSG y PG (en conjunto) en niños antecedentes de SAOS encontrándose un valor predictivo positivo de $97 \% \mathrm{y}$ negativo de $47 \%$; es decir es útil como método de screaning, sin embargo, un resultado normal no descarta un TRS ${ }^{14}$. Se requieren estudios adicionales que confirmen estos datos. 

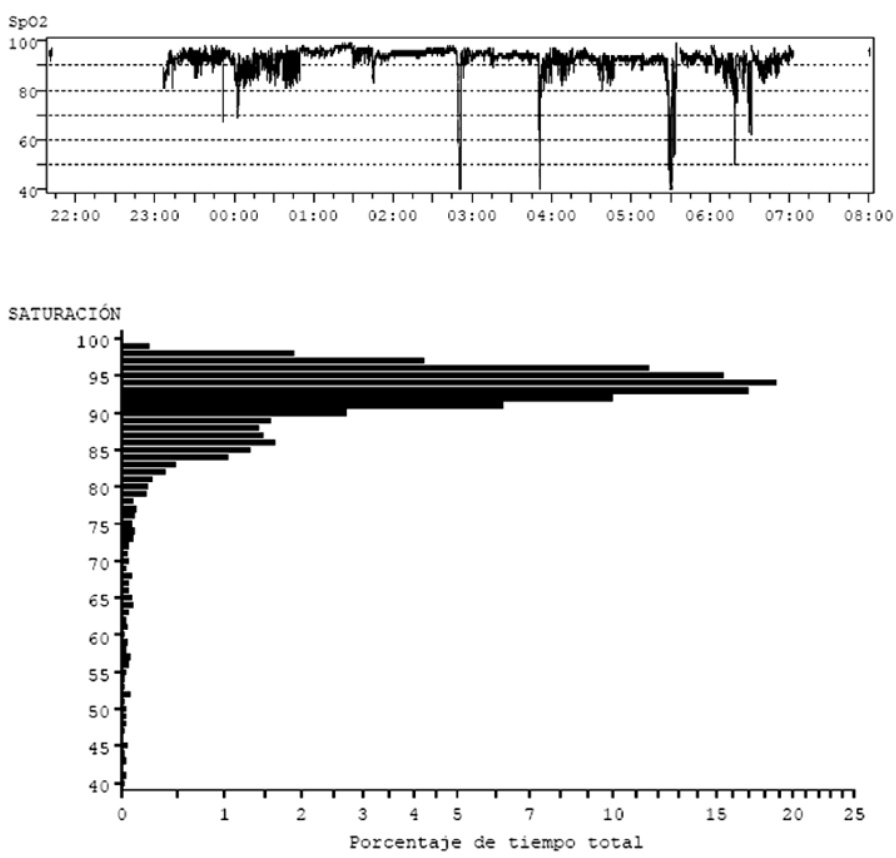

Figura 3. Saturometría continua alterada. Se observan desaturaciones frecuentes, $\mathrm{SaO}_{2}<90 \%$ por $>10 \%$ del TTS.

\section{Estudios diurnos}

En este grupo se incluyen distintos estudios que indirectamente nos sugieren presencia de TRS. Como característica general de ellos es que se realizan en vigilia y que su alteración muchas veces, esta presente en estadios clínicamente evidentes de una patología, como en las ENM, que secundariamente provocan alteraciones en sueño ${ }^{11,25}$.

\section{a. Gases en sangre}

Los gases en sangre nos permiten establecer el diagnóstico objetivo insuficiencia respiratoria global o parcial; constituyen un examen básico, disponible en la mayoría de los hospitales, sin embargo, se alteran en etapas avanzadas de TRS. Una gasometría normal no descarta TRS. La presencia de $\mathrm{PaCO}_{2}$ en vigilia $>50 \mathrm{mmHg}$ asociada a exceso de base $>$ a $4 \mathrm{mEq} / \mathrm{L}$ se relaciona con hipoventilación nocturna, sobre todo en pacientes con distrofia muscular de Duchenne ${ }^{24}$. La correlación entre el aumento de la $\mathrm{PaCO}_{2}$ y de la disminución de la capacidad vital forzada (CVF) es mayor que la existente entre la CVF y el tiempo de registro con $\mathrm{SpO}_{2}<90 \%{ }^{20,25}$.

\section{b. Fuerza muscular respiratoria}

La evaluación de la capacidad muscular inspiratoria global, se realiza a través del registro de la presión ins- piratoria máxima en boca (Pimax). Es un examen no invasivo, que no se realiza frecuentemente en la práctica clínica, sin embargo, la evidencia actual sugiere que su empleo es útil para la evaluación y seguimiento de pacientes con patologías primarias y secundarias de la bomba respiratoria; en quienes se planifican estrategias como la asistencia ventilatoria no invasiva y el entrenamiento muscular respiratorio específico con válvulas de umbral regulable. Dentro de sus ventajas destaca su fácil implementación, bajo costo y valores de referencia confiables para niños y adolescentes $^{26,27}$.

El inicio de TRS se ha asociado a Pimax $<45 \mathrm{~cm} \mathrm{H}_{2} \mathrm{O}$ (Sensibilidad 82\%, Especificidad 89\%), hipoventilación alveolar continua cuando la Pimax $<40 \quad \mathrm{~cm} \mathrm{H}_{2} \mathrm{O}$ (Sensibilidad 95\%, Especificidad $65 \%$ ) y síntomas diurnos severos con Pimax $<35 \mathrm{~cm} \mathrm{H}_{2} \mathrm{O}$ (Sensibilidad 92\%, Especificidad 55\% ${ }^{28}$.

\section{c. Espirometría}

Constituye un examen básico de un laboratorio de función pulmonar, actualmente disponible en atención primaria de nuestro país. La CVF se ha relacionado con TRS en pacientes con ENM; una disminución progresiva de la CVF se relaciona progresivamente con trastornos en sueño REM, no REM y síntomas diurnos que condicionan finalmente una falla respiratoria severa ${ }^{28}$.

El inicio de TRS se asocia con CVF $<60 \%$ (Sensibilidad 91\%, Especificidad 89\%), hipoventilación nocturna cuando la CVF < 40\% (Sensibilidad 94\%, Especificidad $79 \%$ ) y síntomas diurnos severos, más hipercapnia en vigilia, con CVF $<25 \%$ (Sensibilidad $92 \%$, Especificidad 93\% $)^{29-31}$. 


\section{d. Capnografia}

La medición de $\mathrm{CO}_{2}$ exhalado $\left(\mathrm{ETCO}_{2}\right)$ y de $\mathrm{CO}_{2}$ transcutáneo $\left(\mathrm{TcPCO}_{2}\right)$ se encuentran altamente correlacionados y son considerados predictores eficaces para determinar en forma no invasiva los niveles de $\mathrm{PaCO}_{2}{ }^{6,9,15}$. Se han empleado en pacientes sanos, con enfermedades respiratorias, trastornos del sueño y usuarios de soporte ventilatorio ${ }^{8,10}$. La medición de $\mathrm{ETCO}_{2}$, es la más frecuentemente empleada, mediante interfases nasobucales, ramas exhalatoria de ventiladores mecánicos o directamente al tubo endotraqueal; la $\mathrm{TcPCO}_{2}$ tiene indicación en aquellos pacientes en los cuales sea difícil obtener $\mathrm{ETCO}_{2}$, como en los lactantes o en situación de obstrucción nasal ${ }^{31}$. La diferencia entre la $\mathrm{PaCO}_{2}$, y estos, generalmente es menor a $5 \mathrm{mmHg}$ y rara vez excede los 10 $\mathrm{mmHg}$. Diferencias mayores se observan frecuentemente en patologías con aumento de la resistencia de la vía aérea periférica que impide lograr el plateau necesario para un apropiado registro de la $\mathrm{ETCO}_{2}{ }^{13,31}$.

Como se menciono previamente, la capnometría puede ser de gran utilidad al incorporarse a los canales de la PSG y/o capnometría, como registro continuo nocturno expresado como porcentaje del tiempo total de registro con $\mathrm{PCO}_{2}>50 \mathrm{mmHg}$. Sin embargo, la medición aislada de la $\mathrm{ETCO}_{2}$, idealmente al despertar, puede servir como un predictor no invasivo de hipoventilación nocturna ${ }^{25,30}$.

\section{Estudios dirigidos a niños con necesidades especiales}

Más que métodos diagnósticos propiamente tal, son de utilidad principalmente para el seguimiento de pacientes con condiciones clínicas específicas. Incluyen evaluaciones que pueden ser realizadas con aplicaciones tecnológicas de determinados equipos, mediante un sistema de "tarjetas de memoria".

\section{a. Lectura de tarjetas de memoria de ventiladores mecánicos:}

Estas tarjetas pueden ser empleadas en pacientes que utilicen generadores de flujo de diversas tecnología, capturando la información referida al patrón respiratorio e interacción paciente-equipo durante la asistencia ventilatoria no invasiva. De esta forma constituyen una herramienta de ayuda para la monitorización y supervisión, considerando el correcto uso de los dispositivos e interfases a través de la interpretación de estos registros, ya sea durante las hospitalizaciones o en el domicilio. Asiste en las decisiones clínicas de titulación de los equipos y cuando es necesario modificar los parámetros ventilatorios durante el seguimiento, como también en monitorizar la adherencia a las indicaciones, crear informes de cumplimiento y realizar análisis cruzado de los datos de distintos pacientes ${ }^{32}$.

Algunos generadores de flujo de presión binivelada, cuentan con una tarjeta de memoria que permite realizar la lectura resumida y pormenorizada del registro de ventilación de los pacientes (figura 4). Esta tarjeta registra la fecha, la hora y la duración de cada sesión, además almacena los datos recogidos en los 6 meses previos, correspondientes a las tendencias de las presiones de IPAP, EPAP, el volumen corriente, la frecuencia respiratoria, el porcentaje de respiraciones activadas por el paciente, la ventilación minuto, las fugas y las alarmas activadas. Pesquisa posibles condiciones subdiagnosticadas por falta de sintomatología clínica, como por ejemplo, ausencia o pobre gatillo del disparo del Bi-nivel (terapia efectiva sólo como Cpap), escape de flujo excesivo, hipoventilación a través de comprobación de volumen corriente exhalado muy bajo y otras vinculadas a TRS que pueden ser registradas simultáneamente con poligrafía (apneas, hipopneas, desaturación ${ }^{20,32}$. La información de apneas e hipopneas, puede corresponder a una interfase mal adaptada y aumento del flujo de escape, más que a una disminución del flujo relacionada a eventos respiratorios verdaderos y por lo tanto, no se recomienda su lectura.

\section{b. Lectura de tarjetas de memoria de monitores de apnea}

Estas tarjetas pueden emplearse en pacientes con indicación de monitoreo domiciliario, como es el caso de niños con eventos aparentemente letales (EAL) de riesgo o EAL mayor ${ }^{33}$.

Si bien el uso de monitores de apnea es 


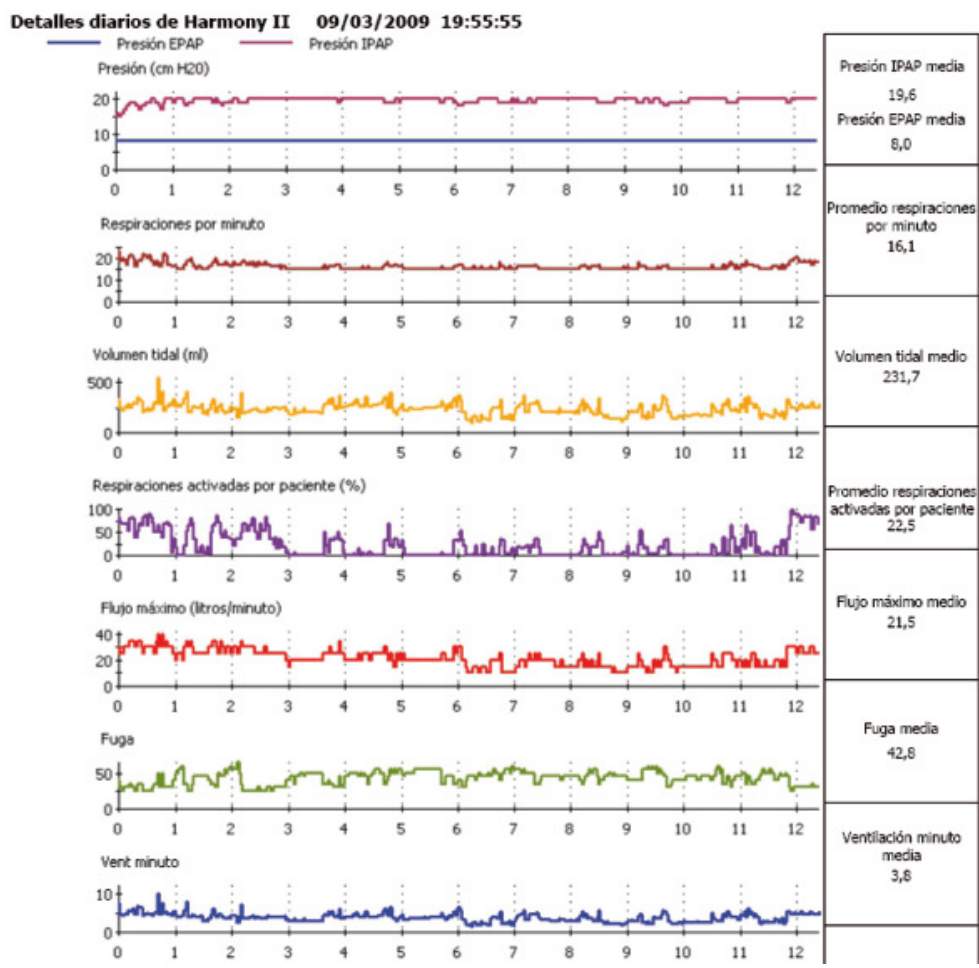

Figura 4. Lectura pormenorizada de tarjeta de memoria de generador de flujo (Bipap Harmony, Respironics-Phillips). controvertido, no ha demostrado una disminución del Síndrome de Muerte Súbita del Lactante y son de poca utilidad en casos de apneas obstructivas; su utilización debe ser evaluada caso a caso, luego de estudios pertinentes por un equipo multidisciplinario. También debe adecuarse en detalle a la situación psicosocial de padres y familia ${ }^{34}$.

Actualmente los monitores más recomendados son los cardiorrespiratorios con tarjetas de memoria, estos deben ser leídos con periodicidad preestablecida (mínimo cada 15 días) o antes en casos de alarmas. El confirmar la presencia de apneas, puede orientar a realizar estudios adicionales. Se han descrito patrones que pueden orientar a determinados diagnósticos, dentro de estos destacan patrones: vasomotores (presentes en apneas emotivas), convulsivos y sugerentes de maltrato infantil ${ }^{35}$.

\section{Conclusión}

La frecuencia de los TRS y el impacto que estos generan en la salud de niños y adoles- centes, en un período altamente vulnerable del desarrollo neurológico y de otros órganos blancos; hace necesario un enfoque pediátrico práctico para el estudio de los TRS. Si bien el examen de elección es la polisomnografía, existen alternativas considerando las características clínicas del paciente, la disponibilidad de estas herramientas en distintos centros asistenciales y la capacitación de los equipos de salud. La poligrafía posee bastante proyección y la saturometría nocturna es una buena alternativa de screaning, ambas tienen la ventaja de realizarse en forma ambulatoria; por lo tanto, de son de menor costo y mayor potencial de cobertura para población pediátrica con factores de riesgo. Los estudios diurnos nos sugieren TRS asociado a hipoventilación, son generalmente más accesibles y recomendados especialmente para pacientes con antecedentes de ENM. El análisis de tarjetas de memoria en niños con necesidades especiales (usuarios de AVNI y/o monitor cardiorrespiratorio) son de especial utilidad para el seguimiento de estos pacientes. 


\section{Referencias}

1.- Marcus $C L$ : Sleep-disordered breathing in children. Am J Respir Crit Care Med 2001; 164: 16-30.

2.- Sociedad Española de Neumología y Cirugía Torácica (SEPAR). Grupo Español de Sueño (GES): Consenso Nacional sobre el síndrome de apneas-hipopneas del sueño. Arch Bronconeumol 2005; 41 Supl 4: 3-4

3.- Marcus CL, Lutz J, Carroll JL, Bamford O: Arousal and ventilatory responses during sleep in children with obstructive sleep apnea. J Appl Physiol 1998; 84: 1926 36.

4.- Brockmann P, Prado F: Estudio de los trastornos respiratorios del sueño en pacientes con enfermedades neuromusculares. Revista Neumología Pediátrica 2008; 3 (Supl): 18-24.

5.- Sans-Capdevila O, Gozal D: Neurobiological consequences of sleep apnea syndrome in children. Rev Neurol 2008; 47: 659-64.

6.- Montgomery-Downs HE, O'Brien LM, Holbrook CR, Gozal D: Snoring and sleep-disordered breathing in young children: subjective and objective correlates. Sleep 2004; 27: 87-94.

7.- Gozal D, O'Brien L, Row $B W$ : Consequences of snoring and sleep disordered breathing in children. Pediatr Pulmonol Suppl 2004; 26: 166-8.

8.- Mellies U, Dohna-Schwake C, Voit T: Respiratory function assessment and intervention in neuromuscular disorders. Curr Opin Neurol 2005; 18: 543-7.

9.- Dayyat E, Kheirandish-Gozal L, Gozal D: Childhood Obstructive Sleep Apnea: One or Two Distinct Disease Entities.? Sleep Med Clin 2007; 2: 433-44.

10.- Gozal D, O'Brien LM: Snoring and obstructive sleep apnoea in children: why should we treat? Paediatr Respir Rev 2004; 5 Suppl A: S371-6.

11.- Katz SL: Assessment of sleep-disordered breathing in pediatric neuromuscular diseases. Pediatrics 2009; 123: S222-5.

12.- American Thoracic Society: Standards and indications for cardiopulmonary sleep studies in children. Am J Respir Crit Care Med 1996; 153: 866-78.

13.- Moss D, Urschitz M, Von Bodman A, et al: Referent Values for Nocturnal Home Polysomnography in Primary Schoolchildren. Pediatr Res 2005; 58: 958-65.

14.- Brouillette RT, Morielli A, Leimanis A, Waters KA, Luciano R, Ducharme FM: Nocturnal pulse oximetry as an abbreviated testing modality for pediatric obstructive sleep apnea. Pediatrics 2000; 105: 405-12.

15.- Kheirandish-Gozal L: Practical aspects of scoring sleep in children. Paediatr Resp Rev 2006; 7S: S50-S54
16.- Iber C, Ancoli-Israel S, Chesson A, Quan SF: American Academy of Sleep Medicine. The AASM Manual for the Scoring of Sleep and Associated Events: Rules, Terminology and Technical Specifications. Westchester: American Academy of Sleep Medicine; 2007.

17.- Wagner $M$, Torrez D: Interpretation of the Polysomnogram in Children. Otolaryngol Clin N Am 2007; 40: 745-59

18.- Marcus $C L$, Omlin KJ, Basinki DJ, et al: Normal polysomnographic values for children and adolescents. Am Rev Respir Dis 1992; 146: 1235-9.

19.- Sánchez-Armengol A, Ruiz-García A, Carmona-Bernal $C$, et al: Clinical and polygraphic evolution of sleep related breathing disorders in adolescents. Eur Respir J 2008; 32: 1016-22.

20.- Waters $K$ : Interventions in the paediatric sleep laboratory: The use and titration of respiratory support therapies. Paediatr Respir Rev 2008; 9: 181-92.

21.- Tonelli de Oliveira AC, Martínez D, Vasconcelos $L F$, et al: Diagnosis of obstructive sleep apnea síndrome and its outcomes with home portable monitoring. Chest 2009; 135: 330-6.

22.- Alonso ML, Terán J, Cordero JA, et al: Reliability of respiratory polygraphy for the diagnosis of sleep apneahypopnea syndrome in children. Arch Bronconeumol. 2008; 44: 318-23.

23.- Sola A, Chow L, Rogido M: Pulse oximetry neonatal care in 2005. A comprehensive state of the art review. An Pediatr 2005; 62: 266-81.

24.- Salyer J: Neonatal And Pediatric Pulse Oximetry. Respir Care 2003; 48: 386-98.

25.- Hukins CA, Hillman D: Daytime predictors of sleep hypoventilation in Duchenne muscular dystrophy. Am J Respir Crit Care Med 2000; 161: 166-70.

26.- Zenteno D, Puppo H, Vera R, et al: Guías de rehabilitación para niños con enfermedades respiratorias crónicas. Neumol Pediatr 2008; 3 (Supl): 25-33.

27.- Szeinberg A, Marcotte JE, Roizin H, et al: Normal values of maximal inspiratory and expiratory pressures with a portable apparatus in children, adolescents, and young adults. Pediatr Pulmonol 1987; 3: 255-8.

28.- Ragette R, Mellies U, Schwake C, Voit T, Teschler H: Patterns and predictors of sleep disordered breathing in primary myopathies. Thorax 2002; 57: 724-8.

29.- Simonds $A K$ : Recent advances in respiratory care for neuromuscular disease. Chest 2006; 130: 1879-86.

30.- Mellies U, Ragette R, Schwake C, Boehm H, Voit T, Teschler H: Daytime predictors of sleep disordered breathing in children and adolescents with neuromuscular disorders Neuromuscul Disord. 2003; 13: 123-8. 
31.- Redline S, Budhiraja R, Kapur V, et al: The scoring of respiratory events in sleep: Reliability and validity. J Clin Sleep Med 2007; 3: 162-200.

32.- Rabec CA, Reybet-Degat $O$, Bonniaud P, Fanton A, Camus $P$ : Leak monitoring in noninvasive ventilation. Arch Bronconeumol 2004; 40: 508-17.

33.- Asociación Latinoamericana de Pediatría: Comité en síndrome de muerte súbita del lactante. Guía de práctica clínica-2005. Episodio de posible amenaza a la vidaALTE.

34.- Zenteno D: Eventos de aparente amenaza a la vida (ALTE): Conceptos, etiología y manejo. Neumol Pediatr 2006; $1: 133-8$.

35.- Poets $C$ : Apparent life-threatening events and sudden infant death on a monitor. Paediatr Resp Rev 2004; 5: S383-6. 